\title{
Acanthosis nigricans at sites of insulin injection in a man with diabetes
}

\author{
Yuanshen Huang MD PhD, Morvarid Hessami-Booshehri MD
}

Cite as: CMAJ 2018 November 26;190:E1390. doi: 10.1503/cmaj.180705

A

69-year-old man with long-standing type 2 diabetes presented with a 2-year history of developing brown lesions on his abdomen and right thigh. He had received insulin injections subcutaneously at these sites for the past 15 years. Individual lesions started small, enlarged progressively and finally coalesced. They were largely asymptomatic. He was otherwise well. Physical examination showed brown, velvety verrucous plaques on the patient's lower abdomen (Figure $1 \mathrm{~A}$ ) and right thigh. We also noted brown verrucous plaques on his knuckles, axillae and groin, but to a lesser degree. Screening for malignant tumour was negative. Glycosylated hemoglobin was $8.8 \%$ (normal level: < 7.0\%). Biopsy of a lesion (Appendix 1, available at www.cmaj.ca/lookup/suppl/ doi:10.1503/cmaj.180705/-/DC1) showed marked compact hyperkeratosis, acanthosis and papillomatosis in the patient's epidermis. We diagnosed acanthosis nigricans and began intralesional steroid injection and topical clobetasol $0.05 \%$ cream, given the presence of lichenification. We also instructed the patient to alternate injection sites and improve his diabetes control. His lesions regressed over 1 year (Figure 1B).

Acanthosis nigricans is characterized by skin darkening and thickening, commonly affecting skin folds (e.g., neck, axillae and groin). Causes of acanthosis nigricans include malignant tumours, endocrinopathies, genetic conditions and certain medications (e.g., oral contraceptives, niacin and systemic corticosteroids). ${ }^{1,2}$ Exogenous drug use, such as insulin injections, is one of the causes and is thought to result from activation of insulin-like growth factor $1 .{ }^{3}$ In our patient, in addition to appearing on the skin folds, the lesions were more pronounced at sites of insulin injection, indicating the local stimulatory growth effect of insulin injections.

The diagnosis of acanthosis nigricans is usually clinical. Differential diagnoses include inflammatory dermatoses, Becker nevus, confluent and reticulated papillomatosis and Addison disease. ${ }^{2}$ Treatment of acanthosis nigricans depends largely on the cause. Correcting the underlying cause might restore the normal skin colour and texture. Other treatment options include topical agents (e.g., tretinoin, urea and salicylic acid) and laser therapy. ${ }^{1}$

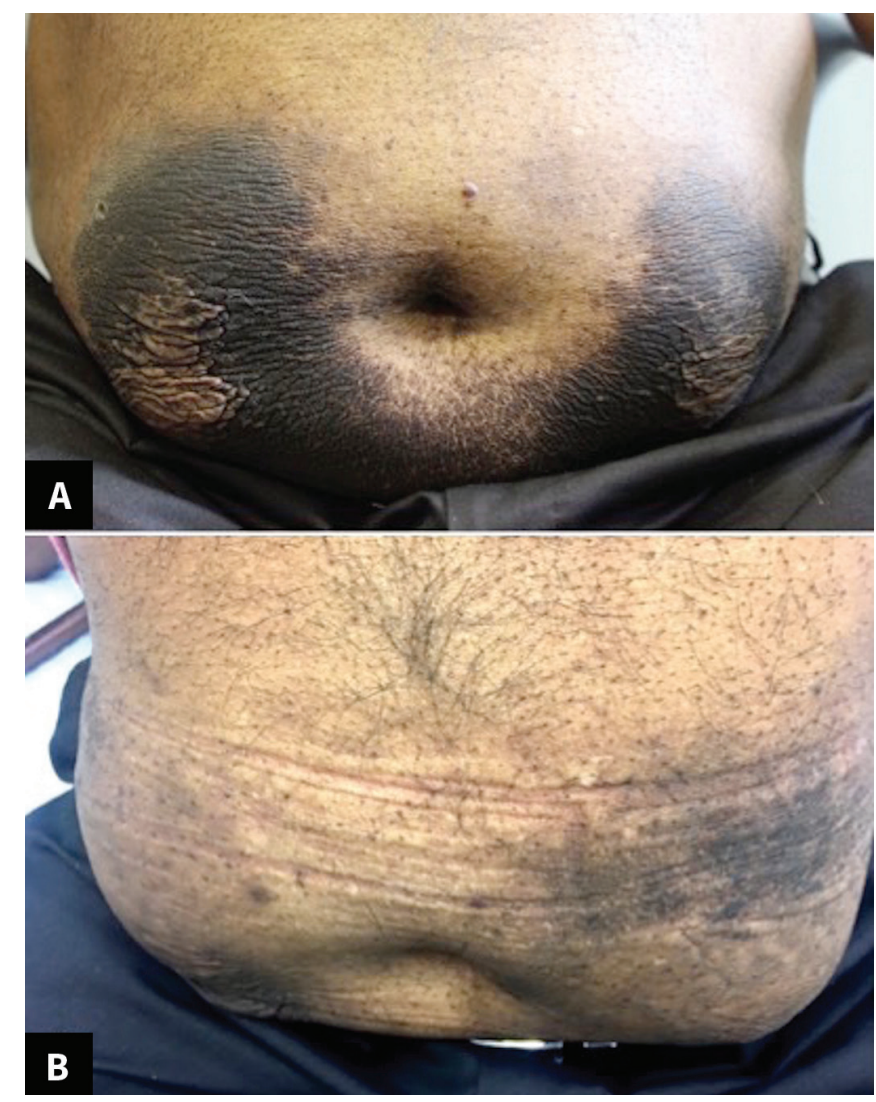

Figure 1: (A) Brown verrucous plaques at sites of subcutaneous insulin injection on the lower abdomen of a 69-year-old man. (B) Clinical improvement after treatment over 1 year.

\section{References}

1. Kutlubay Z, Engin B, Bairamov O, et al. Acanthosis nigricans: a fold (intertriginous) dermatosis. Clin Dermatol 2015;33:466-70.

2. Sinha S, Schwartz RA. Juvenile acanthosis nigricans. J Am Acad Dermatol 2007;57:502-8.

3. Yahagi $\mathrm{E}$, Mabuchi T, Nuruki $\mathrm{H}$, et al. Case of exogenous insulin-derived acanthosis nigricans caused by insulin injections. Tokai J Exp Clin Med 2014;39:5-9.
Competing interests: None declared.

This article has been peer reviewed.

The authors have obtained patient consent.
Affiliations: Division of Dermatology (Huang), Department of Medicine, University of Toronto, Toronto, Ont.; Scarborough General Hospital (Hessami-Booshehri), Scarborough, Ont.
Correspondence to: Yuanshen Huang, yuanshen.huang@mail.utoronto.ca 